\title{
Adaptive all the way down: Building responsive materials from hierarchies of chemomechanical feedback
}

\section{Citation}

Grinthal, Alison, and Joanna Aizenberg. 2013. "Adaptive All the Way down: Building Responsive Materials from Hierarchies of Chemomechanical Feedback." Chemical Society Reviews 42 (17): 7072. doi:10.1039/c3cs60045a.

\section{Published Version}

doi:10.1039/c3cs60045a

\section{Permanent link}

http://nrs.harvard.edu/urn-3:HUL.InstRepos:37255476

\section{Terms of Use}

This article was downloaded from Harvard University's DASH repository, and is made available under the terms and conditions applicable to Open Access Policy Articles, as set forth at http:// nrs.harvard.edu/urn-3:HUL.InstRepos:dash.current.terms-of-use\#OAP

\section{Share Your Story}

The Harvard community has made this article openly available.

Please share how this access benefits you. Submit a story.

\section{Accessibility}




\title{
Adaptive all the way down: Building responsive materials from hierarchies of chemomechanical feedback
}

\author{
Alison Grinthal $^{1}$ and Joanna Aizenberg ${ }^{1,2,3,4}$ \\ ${ }^{1}$ School of Engineering and Applied Sciences, Harvard University, United States \\ ${ }^{2}$ Wyss Institute for Biologically Inspired Engineering, United States \\ ${ }^{3}$ Department of Chemistry and Chemical Biology, Harvard University, United States \\ ${ }^{4}$ Kavli Institute for Bionano Science and Technology, United States
}

\begin{abstract}
A living organism is a bundle of dynamic, integrated adaptive processes: not only does it continuously respond to constant changes in temperature, sunlight, nutrients, and other features of its environment, but it does so by coordinating hierarchies of feedback among cells, tissues, organs, and networks all continuously adapting to each other. At the root of it all is one of the most fundamental adaptive processes: the constant tug of war between chemistry and mechanics that interweaves chemical signals with endless reconfigurations of macromolecules, fibers, meshworks, and membranes. In this tutorial we explore how such chemomechanical feedback - as an inherently dynamic, iterative process connecting size and time scales - can and has been similarly evoked in synthetic materials to produce a fascinating diversity of complex multiscale responsive behaviors. We discuss how chemical kinetics and architecture can be designed to generate stimulus-induced 3D spatiotemporal waves and topographic patterns within a single bulk material, and how feedback between interior dynamics and surface-wide instabilities can further generate higher order buckling and wrinkling patterns. Building on these phenomena, we show how yet higher levels of feedback and spatiotemporal complexity can be programmed into hybrid materials, and how these mechanisms allow hybrid materials to be further integrated into multicompartmental systems capable of hierarchical chemo-mechanochemical feedback responses. These responses no doubt represent only a small sample of the chemomechanical feedback behaviors waiting to be discovered in synthetic materials, and enable us to envision nearly limitless possibilities for designing multiresponsive, multifunctional, self-adapting materials and systems.
\end{abstract}

\section{Introduction}

The nonstop beating and whirling of living systems are nature's answer to, as Szent-Gyorgyi put it, an electron looking for a place to rest. By sequestering chemical species in confined microarchitectures, living materials set the stage for energetically coupled reaction cascades that would likely be unfavorable anywhere else. But the reaction components do far more than simply mingle inside a passive architecture. Rather, they are the architecture: reactants and catalysts are strung together in long chains, twisted into filaments, woven into networks, and packed into bilayers, with all of them wrapped around each other in a dense, interconnected jungle. Even "freely diffusing" molecules are hardly free, as membranes and fibrous meshes herd them into localized concentrations and gradients. The result is a dynamic meta-reaction chamber 
that actively positions reaction components, orients bonds, and channels chemical reactions into spatiotemporally controlled relays. Yet building the architecture out of reaction components comes with a catch. At the nano- and microscales, chains and networks reconfigure according to local bond energies but also answer collectively to the laws of mechanics, which introduce an entirely different definition of "rest". Thus as reactions proceed, entire fibers bend and twist, meshworks expand and contract, membranes stretch and pucker - in ways the electrons wouldn't necessarily dictate - as they accumulate and seek to relieve mechanical stress. Stress-driven motions in turn rearrange the reaction components, and a complex multiscale feedback dance ensues as the system's chemical and mechanical elements constantly adjust to each other.

The electron may not appear to be getting much rest, but the organisms are doing quite well. All of this adapting built into the fabric of their most basic materials is exactly what makes the organisms themselves so adaptive. It not only keeps them on their toes in responding and adapting to their constantly changing environment, but also enables each organism to function as an integrated system of cells, tissues, and networks all continuously adapting to each other. The iterative, step-by-step nature of the chemomechanical feedback allows both chemical pathways and multiscale motions to be rewired in nearly infinite different combinations in response to different stimuli. Within cells alone, heat, pressure, voltage, $\mathrm{pH}$, or any number of molecular signals can reorchestrate the same cellular contents into distinct chemomechanical repertoires - bond formation and cleavage, ion and molecular transport, interspersed with filaments pushing and pulling, extensions shooting out and retracting, and appendages waving - leading cells to divide, migrate, differentiate, aggregate, or die as appropriate. Likewise, the feedback repertoires in different cells can be intercalated with each other in endlessly different combinations, leading to hearts, lungs, and nerves that beat, heave, and fire in complex stimulus-triggered spatiotemporal patterns. All put together, we meet the outside world with multiresponsive, multifunctional skin that opens pores and sweats in the heat, fluffs up hairs in the cold, darkens protectively in bright sun, senses localized pressure, heals its own wounds, and signals various forms of internal distress by turning blue, yellow, or red.

Not quite so our houses, cars, planes, and other constructions. The need for responsive materials is increasingly recognized as key to the future of everything from energy-efficient buildings to airplane safety to targeted medicine. But our ability to design sophisticated adaptive systems faces several fundamental materials challenges: how do we make a material dynamic; how do we program stimulus sensitivity; how do we generate complex and diverse spatiotemporal patterns; how do we make a material multifunctional; and how do we integrate it with others as a bottom-up building material into fully adaptive systems. The critical insight from biological systems is that these questions all boil down to the same answer. As inherently adaptive and multiscale, chemomechanical feedback unites all of them with the same underlying mechanism. The question thus becomes how - or even whether - synthetic nano/microarchitectures and chemistries can be designed and combined to produce anywhere near the adaptive complexity the living systems tempt us to envision. The answer, it turns out, may lie not in looking to biology as a model for designing new synthetic materials from scratch, but in using it as a unifying lens for considering and building on the potential for chemomechanical feedback in the synthetic materials we already have. 
In this review, we explore the myriad ways feedback between chemistry and motion has already been harnessed to produce complex dynamically evolving 3D patterns in synthetic systems. While many of the approaches have been developed specifically for their responsive potential, some blur the line between fabrication and responsive strategies. Our aim in examining them collectively is 1) to develop a unified map of the multiscale "adaptive space" achievable in synthetic materials, and 2) to discover both common and complementary elements that potentially provide a basis for crosspollinating and synthesizing disparate lines of work into comprehensive, hierarchical adaptive systems. We start by looking at how chemical kinetics and architecture can be combined to evoke a rich terrain of spatial and temporal patterns within a single bulk material. We then add to this an entirely different, complementary class of dynamic surface patterns that emerges specifically from chemomechanical feedback between the material's interior and its interface. Building from both of these, we show how composite materials - effectively a collection of many such interfaces arrayed within a common matrix - provide yet another hierarchical level of feedback and dynamic complexity. Finally, we present mechanisms for integrating these processes into hierarchical compartmentalized materials systems displaying higher order chemical and mechanical feedback, both internally and with the surrounding environment.

\section{Reactions, diffusion, and stress in a polymer network}

While in principle all materials combine chemistry and mechanics, one major class - polymeric materials - has been shown to display particularly versatile chemomechanical behavior $^{1-3}$. Like the macromolecules that fill living cells, synthetic polymer chains pack into networks that selectively allow solvent and other chemical species to diffuse in and out, allowing a broad assortment of chemical reactions to take place within them. Reactions can directly modify the polymer chains, alter their interactions, or involve solution-based processes in the interchain space. Any of these can in turn alter the network's propensity to absorb solvent, generally by altering the osmotic pressure or the chains' affinity for solvent and/or each other. Either way, solvent uptake is a two-way street: as the solvent diffuses into the network, the network (also known as a hydrogel if the solvent is water) diffuses into the solvent ${ }^{4}$ - that is, parts of the network move relative to each other, leading to size and shape changes. Herein lies the root of its chemomechanical behavior. While the polymer network diffuses like a chemical species, at the same time its diffusion coefficient contains its elastic modulus in place of the thermal term kT. Diffusion thus incurs mechanical stress, so that reactions, diffusion, and $3 \mathrm{D}$ elastic reconfiguration are inextricably linked at the crossroads of chemical and mechanical forces.

\subsection{Chemistry as a mover and sculptor}

This intimate connection enables polymeric materials to translate the detailed kinetics of chemical flows, gradients, and reactions into complex nano/microscale shapes, patterns, and motions. The potential diversity and tunability of this coupling are exemplified by a wide-ranging collection of topographic micropatterns created by reaction-diffusion processes in polymer films ${ }^{5,6}$. When an initiating reagent is introduced 
into a film uniformly doped with a second reagent, the film begins to morph into complex topographies as the reagents diffuse and react and the product drives local water uptake. The evolving topography captures the dynamic spatiotemporal pathways of the reaction, developing primary and secondary sets of ridges in synchrony with primary and secondary chemical diffusion fronts ${ }^{5}$ (Figure 1A). Different chemical combinations, with different kinetics and associated swelling, each produce signature patterns. The sensitivity of chemomechanical coupling is further demonstrated by the radically different, multi-tiered topographic patterns formed when the initiating reagent is presented in different 2D geometries that alter reaction propagation. The degree of swelling is directly proportional to local product formation, and the features' shape, spacing, height, slope, and width can each be finely tuned by varying not only the reagent concentrations but also the elasticity, as set by the polymer crosslinking density ${ }^{6}$ (Figure 1B). And of course, the concentrations, crosslinking density, and other factors that influence reaction, diffusion, and reconfiguration all change locally and dynamically as the film swells.

In these examples, pattern formation is irreversible, so that dynamic progression leads to increasingly complex, superimposed spatial details. But with other reactions, the chemical pathways can be captured as dynamic 3D waves propagating across the film. This has been elaborately demonstrated for the Belousov-Zhabotinsky (BZ) reaction, a classic oscillatory reaction ${ }^{7,8}$. In this case, catalyst molecules are directly bound to the polymer, and the reaction is initiated by introducing the reagents into the network. As the reagents diffuse and react, the catalyst is transiently oxidized and reduced. Since the oxidized catalyst - and hence the attached polymer - is more hydrophilic than the reduced form, or, in some cases, vice versa ${ }^{9}$, the network locally absorbs and releases water as the propagating reaction switches the redox state back and forth. The changing topography thus tracks when, where, and to what extent the reaction is taking place, with features growing, shrinking, and changing shape ${ }^{7,8,10}$ as reaction fronts pass through (Figure 1C). The period, amplitude, and other features of the topographic waves are, as above, acutely sensitive to reagent concentration and crosslinking density - which, in turn, change with local swelling and deswelling of the network. This two-way feedback has been shown to introduce complex kinetic features both experimentally and in computational models of the system ${ }^{8,11}$.

\subsection{Linking to stimuli}

In addition to these reactions, an ever-growing assortment of polymer chemistries has been developed to couple network mechanics with particular stimuli. These introduce not only a diversity of sensitivities - to $\mathrm{pH}^{12}$, temperature ${ }^{13,14}$ light $^{15,16}$, biomolecules ${ }^{17}$, magnetic and electric fields ${ }^{18}$, pressure $^{19}$, and redox state - but also a diversity of mechanisms for propagating the response and interweaving chemical kinetics and diffusion with mechanical stress and reconfiguration (Figure 2). For example, a pHsensitive network modified with acidic functional groups undergoes proton dissociation at high $\mathrm{pH}$, leading to a multi-species diffusion/reaction cascade of $\mathrm{H}^{+}$combining with $\mathrm{OH}^{-}$, cations and $\mathrm{OH}^{-}$entering, and osmotically-driven water uptake and network expansion $^{12}$. A glucose-responsive network modified with glucose-binding moieties also involves diffusion and binding steps, but reconfiguration is driven by the complex charge 
equilibrium on the chains themselves; the increase in both free and glucose-bound hydrophilic groups directly induces mixing with water ${ }^{17}$. Temperature-responsive networks involve no signal diffusion, but the chain-based response drives mechanical reconfiguration by a combination of increased affinity for water and decreased chain interactions ${ }^{20}$. Light responsive mechanisms, which come in many forms, have been integrated with temperature- and $\mathrm{pH}$-responsive modifications to produce both additive and cooperative multiresponse pathways that rely on combinations of charge diffusion, solvent affinity, and chain interactions that are each highly tunable and entail multiple forms of chemical-mechanical interfacing ${ }^{15}$.

Although these response pathways have not been designed specifically for topographic patterning, they too appear to contain the necessary ingredients for producing complex chemomechanical feedback behaviors and spatiotemporal responses. The kinetics of the glucose response, for example, generates a chemomechanical instability that creates a spatially and temporally heterogeneous swelling process ${ }^{17}$. Since hydration of the polymer chains is slower than the diffusion of water into the network, a distinct phase boundary develops at the diffusion front, with an unstable elastic pressure difference between the swelled and nonswelled sides. The phases coexist until, at a critical point, the elastic pressure in the swollen phase triggers expansion of the adjacent region, accompanied by accelerated diffusion. A similar interplay - the formation, stabilization, and breakdown of a spatial discontinuity through chemistry driving mechanics driving further chemistry - has been observed in temperature-responsive networks ${ }^{17}$. The shrinking processes of both systems have also been seen to produce a series of unusual shapes attributed to chemomechanical feedback between spatially discontinuous phases. Dynamic spatial domains also arise in a $\mathrm{pH}$-based system with completely different kinetics, generating spontaneous oscillations purely through the feedback between reactions, diffusion, and mechanical responses ${ }^{21}$.

\subsection{Synergy between architecture and chemistry}

Since chemistry and mechanics evolve in partnership, these feedback processes can be shaped not only by reaction kinetics but also by physical factors that directly control how stress and strain propagate through the material. In the BZ system, local mechanical stress can set off the chemical reaction and send it propagating through the film, by reconfiguring the polymer chains, altering the crosslinking density and local reagent concentrations, and lowering the barrier to solvent entry and species diffusion ${ }^{8}$. Modeling indicates that different stress magnitudes and/or locations produce vastly different spatial domains and patterns of wave propagation (Figure 3A). Local or global stress can likewise induce or alter the chemical behavior of stimulus-responsive materials. Global stress not only changes the transition temperature of a temperatureresponsive network but also introduces a new twist into its chemomechanical crosstalk and the dynamics of spatial segregation ${ }^{22}$. Force-induced displacement of the polymer chains lowers the barrier to solvent diffusion, chain hydration, and swelling - but the resulting swelling then relieves stress in adjacent regions and inhibits further hydration and diffusion. The stress-modified chemomechanical feedback thus generates distinct spatial domains, with boundaries that vary according to stress magnitude (Figure 3B). 
Complex local and global stresses can effectively be programmed into the material's architecture by designing shapes, confinements, and defects that concentrate, localize, and relieve stress unevenly as the network swells. BZ waves have been shown to reverberate in dramatically different patterns within sharp-cornered rectangles, circles, and other geometries depending on aspect ratio and size as well as overall shape ${ }^{8,10}$. Recent theoretical modeling of $\mathrm{pH}$-responsive systems shows that inhomogeneous stress fields - as well as inhomogeneous network displacement, degree of acid dissociation, and solvent and ion concentrations - develop as swelling takes place in a film attached to a rigid substrate, a cylindrical pillar on a base, a sphere on a rigid core, and other confinements $^{23}$. Temperature-sensitive networks similarly develop characteristic stress patterns in a variety of confined geometries, with transition temperatures varying throughout the network according to distance from the rigid substrate ${ }^{24}$. Even more elaborate stress fields have been shown to develop in networks patterned with structural defects; the defects act as sites for stress relief during swelling, leading to uneven deformation and diffusion ${ }^{23}$ (Figure 3C).

\section{Adaptation at the interface}

\subsection{Interior kinetics and surface buckling}

Meanwhile, as these feedback processes play out inside the bulk material, the surface as a whole can constantly adapt to them with a higher level of dynamic wrinkling and buckling responses. A swelling network was first witnessed over two decades ago to proceed through a parade of morphing surface patterns: fine textures emerged, units coalesced, and one distorted honeycomb gave way to another ${ }^{25}$. Rather than reflecting swelling per se, these patterns were attributed to periodic buckling triggered by compressive surface stresses; such stresses can develop from differential degrees of swelling, such as between a confined base and a free surface or between swelled and unswelled sides of a diffusion front. An immense field of surface instability-induced patterning has since characterized many of the mechanical rules for predicting the most stable patterns ${ }^{26,27}$, but in a swelling network the pattern develops iteratively, in tandem with the diffusion front, and is determined kinetically at each step by the front's evolving geometry $^{28-30}$. Diffusion radiating from a central point, for example, can generate concentric wrinkles perpendicular to the diffusion direction even if these curved waves are not the most stable overall. Only later, as the diffusion front's curvature increases, does the surface reach a threshold and start to transform to the more stable herringbone pattern (Figure 4A, left) ${ }^{28}$. Similarly, wrinkles fork, split, and merge as a linear front progresses (Figure 4A, right) ${ }^{28}$.

The iterative adaptation process means that the surface patterns are highly sensitive to diffusion kinetics. If polymer swelling is dominated by Fickian kinetics - in which motion of the solvent front is fast compared to periodic formation of wrinkles solvent diffusing from a central point generates a spoke pattern (Figure 4B, left) ${ }^{29}$. However, if the diffusion front moves more slowly and with a discrete interface - "Case II" kinetics, as in the glucose-responsive system discussed above - the pattern is completely different: a target pattern of concentric circles ${ }^{29}$ (Figure 4B, right). The difference between the spoke and target motifs is due to nothing more than a higher 
degree of polymer crosslinking that slows the swelling response in the latter case, leading to different balances and orientations of the stress fields. But equally dramatic differences can be obtained by varying the strength of the chemical interactions between the solvent and polymer chains, as has been demonstrated by swelling with different types of solvents: a gradation of solvents with high to low mixing energies produces a range of distinct wrinkling motifs, and these can be further modified by varying the crosslinking density $^{31}$. At the same time, the relative swelling and buckling rates are also sensitive to chemical and mechanical features of the interface, such as surface tension of a gas or liquid or stiffness of an overlaid material, that influence the threshold for buckling ${ }^{32}$.

\subsection{Defects and colliding wave fronts}

As in the bulk material, here, too, architecture can synergize with kinetics to generate higher order feedback responses. The dynamic nature of surface evolution allows the patterns to adapt not only to a single changing diffusion front, but also to each other as two propagating patterns meet, merge, and require the surface to recalculate a new balance of stresses (Figure 4C, left). Since diffusion frequently initiates at tiny surface defects, an array of defects can be designed to create a surface full of wrinkling patterns each propagating according to their local diffusion kinetics but ultimately all coming together into a surface-wide collective motif ${ }^{30}$ (Figure $4 \mathrm{C}$, right). The new pattern retains domains, with sizes determined by the distances between defects, but the wrinkling morphologies of the original patterns are completely re-shaped by the complex elastic merging process. The labyrinthine morphology arises from the interplay between diffusion kinetics and architecture, as the Fickian kinetics generate radial spokes and the structural defects shape how the spokes reorient into hierarchically organized parallel lines.

Since surface dynamics are tied to the chemical and mechanical fields within the whole network, they can also be influenced by architectural defects in the base. A raised microfeature on a confining substrate has been shown to create a complex deformation field during swelling: since the region above the step is thinner than the adjacent regions, it swells proportionately less, and the surfaces of the thicker regions expand laterally toward it ${ }^{33}$. As a result, the surface over the step is subject to planar compressive stresses from the neighboring regions (Figure 4D, left). Similarly to the diffusion fronts discussed above, the anisotropic swelling and planar strains generate a surface instability, and the surface regions collectively adapt by buckling to form a crease. A wide defect can induce two creases, while topographically patterning the substrate with an array of raised features creates a set of interacting surface strains that influence the location, spacing, and morphology of surface responses. The mechanically driven surface dynamics have, in turn, been shown to feed back into the network's chemical and diffusion processes. The local polymer crosslinking density is significantly higher over the step - that is, in the creased area - than in the surrounding regions (Figure 4D, right), and is proposed to account for the inhibition of small molecule transport through this part of the network.

\subsection{Bending and twisting}


If we shrink this entire scene down to the microscale and, instead of broad films, consider a high aspect ratio microcylinder with swellable and nonswellable sides (Figure 5 , left), we still see solvent-induced surface instabilities and buckling, but now they take the form of the whole cylinder bending, curling, and twisting into different shapes ${ }^{34}$ (Figure 5, right). In these examples, the cylinders consist of lengthwise compartments composed of either a water-swellable polymer or an inert material. As the polymer chains become hydrated and the compartment expands, the mismatch between its free and attached surfaces generates planar stresses. If the stress at the interface is sufficient to overcome the stiffness of the attached material, the compartments collectively relieve the stress by bending. Since, particularly at the micro/nanoscale, the aspect ratio has a significant impact on stiffness, the cylinder's bending radius is a function of both its initial geometry and the degree of polymer swelling as well as elastic modulus: short cylinders, partial hydration, or swelling of only a quarter of the cylinder each lead to smaller deflections. In addition, since buckling depends on anisotropic forces, varying the symmetry and configuration of the compartments produces a wide range of motions and shapes. A cylinder with equal swelling on both sides doesn't bend at all, while inhomogeneities in the compartmentalization lead to non-uniform bending and twisting (Figure 5, red arrows).

\section{Arrays of microinterfaces inside a common material}

Taking stock, we have 1) a matrix that, when triggered, springs to life in swirling interwoven currents of chemical reactions, diffusion, and elastic deformations, and 2) an interface that responds to these with higher order collective surface motions that feed back and reshape the currents. Dynamic hybrid microstructures remind us that such an interface can take many forms, preserving diversity of motions and sensitivity down to the microscale. This means that, in addition to the matrix-wide external interface, we can create adaptive interfaces anywhere inside the matrix by embedding it with dynamic microstructures analogous to those discussed in Section 3.3. We thus come to the next level of feedback complexity: we can now weave higher order feedback throughout the system by, in essence, spiking the matrix with many local higher order feedback processes - that is, microstructures each integrating their own local patches of chemomechanical dynamics into responsive bends, twists, etc. Together, the structures become a dynamic part of the architecture, acting as defects that influence reactions, diffusion, and mechanical stress but also reconfigure in response to them. At the same time, their motions translate the dynamics of their respective local regions into a collective readout - but reshape and possibly integrate the local dynamics in the process, ultimately mapping them into higher order responses not possible with the matrix alone.

\subsection{Dynamic defects}

A broad assortment of nano/microparticles has been designed expressly to induce, localize, and channel - as well as respond to - reactions, diffusion, and stress in responsive materials ${ }^{35}$ (Figure 6A). Several varieties serve as localized sites for initiating chemical responses in response to stimuli: for example, several types of particles can be triggered to produce heat in response to light, electric, or magnetic signals. The localized 
reactions influence the rate, efficiency, and path of heat transfer to the rest of the matrix, enabling more complex responses than in a bulk responsive matrix ${ }^{35}$. High aspect ratio nanofibers have also been designed to act as localized sites for solvent uptake; fibers that are more hydrophilic than the polymer chains, for instance, act as water channels that guide both the entrance and the internal diffusion pathways of water ${ }^{36}$. Still other particles moderate matrix mechanics. Particles bearing elastic surface molecules covalently bound to the matrix can locally restrain its expansion or deformation, while interconnected stiff high aspect ratio fibers acts as stress transduction networks that redirect stress away from the rest of the matrix and increase its mechanical resistance ${ }^{35}$. Many particles are designed to reversibly reconfigure - in most cases, associate or dissociate - in response to chemical stimuli, solvent uptake, or mechanical stress, altering how or whether they function ${ }^{35-37}$. For example, $\mathrm{pH}$-sensitive nanofibers associate into stress-channeling networks specifically at high $\mathrm{pH}$ and lose their function at low $\mathrm{pH}^{37}$ (Figure 6B).

While these particles are chemically equipped to participate in a wide range of localized feedback processes, few have been designed for sophisticated mechanical sensing and dynamic reconfigurations. In contrast to the aggregation of randomly suspended particles and fibers, oriented, flexible nanofibers have shown an amazing capacity to assemble into diverse, reproducible, and tunable microarchitectures in response to mechanical forces. A "forest" of carbon nanotubes anchored to a substrate, for instance, develops a complex set of lateral and axial forces in an evaporating solvent, driving them to bend collectively into bridges or opening flowers, helices, or opening and closing iris shapes (Figure 7A), to name just a few of the possibilities ${ }^{38}$. The overall shapes and detailed bending profiles can be rationally predicted and are extremely sensitive to the layout of the fibers, as well as to mechanical coupling between the tips and the substrate base and to the amount of slip between assembling fibers.

The dynamic assembly of nano/microscale fibers into complex patterns and architectures is in fact an intricate story of chemomechanical feedback all in itself. A simple, regular array of polymeric fibers in an evaporating solvent evolves step by step into multiscale, hierarchical helices, bundles, and chiral swirls (Figure 7B), shaped at every stage - like the reconfiguring polymer network and the buckling surface - by the opposing pulls of chemistry and mechanics ${ }^{39}$. Fibers bend and twist in response to solvent forces but are elastically driven to return to their upright positions. Molecular attractions between their surfaces, however, drive them to maximize their contact area by wrapping around each other or zipping together - an even worse offense elastically. They thus inch through a series of slips and buckles as their elastic resistance is matched against their surface attractions. The process repeats itself at the next stage as solvent forces bring the resulting bundle - with its new elastic and surface profiles - together with another one. The same hierarchical steps work in reverse, making the pattern subject to disassembly as well as reconfiguration as the balance of forces changes at each stage. The evolving forms are sensitive not only to mechanical forces but also to the chemical nature of the local environment. Fibers coated with self-assembled monolayers (SAMs) of various bonding types and strengths can be selectively dissociated by changes in solvent polarity, etc., and a wide selection of SAMs with redox, $\mathrm{pH}$, light, and other specific switchable interactions has also been developed ${ }^{40}$. 
However the fibers move, their motions in turn feed back into the dynamics of the surrounding medium as the reconfiguring tips resculpt the surface into complex topographies. The fiber patterns and surface patterns thus evolve together through a mutual dynamic feedback process. This exchange is both local and long range: since each tip directly contacts the surface, its influence directly reflects its individual motions. At the same time, the local change in surface contour is felt by the neighboring fibers, so that the force is transmitted to them whether or not they contact each other. This interplay between oriented, anchored fibers and the surface thus introduces another mechanism for higher order feedback and can lead to complex, even chiral long-range patterns ${ }^{39}$ (Figure 7C).

\subsection{Configuring hybrid architectures for feedback}

We now face the rather heady prospect of integrating the chemomechanical feedback dynamics of responsive polymer networks - themselves an integrated world of nested feedback - with the chemomechanical feedback hierarchies of flexible nano/microstructures. Certainly the patterns, motions, and overall responsive repertoires of the system are ultimately shaped by the combinatorial choices of chemical reactivity, kinetics, molecular interactions, and elastic moduli of each component. But the first fundamental task is architectural: how the components are physically configured determines how both mechanical and chemical signals are exchanged between them. In particular, the nanostructures, as part of the architecture, shape how the network can deform and swell as discussed in sections 2 and 3, but at the same time are shaped by its forces through the same interfaces.

Two basic configurations have been developed for integrating anchored arrays of high aspect ratio nanostructures with swelling polymer networks, with distinct modes of force exchange and many variations stemming from each of them. In one, the network is overlaid on the array and is covalently bound to both the substrate base and the sides of the nanostructures ${ }^{41}$ (Figure 8A, top left). With these constraints, the network expands and contracts primarily in the vertical direction, exerting forces that bend and straighten the nanostructures. If the structures are oriented vertically - that is, in the same direction as expansion and contraction - contraction-induced bending incurs elastic strain and generates counteractive forces opposing network contraction. The magnitude of force exerted on each structure is determined by the local swelling ratio, so that swelling inhomogeneities can generate anisotropic forces. Alternatively, using multiphoton lithography, the network layer can be suspended on the nanostructures, parallel to but completely free from the substrate base ${ }^{42}$ (Figure $8 \mathrm{~A}$, top right). In this configuration, expansion and contraction are primarily lateral. Expansion now drives the nanostructures apart from each other, but since they remain attached to the base they bend and incur strain. The force magnitude and direction vary with position relative to the perimeter of the film, so that spatial inhomogeneities are biased a priori by the shape and complexity of the outer edges.

An additional interesting configuration has recently been reported based on the carbon nanotube forests discussed in section $4.1^{43}$. Similar to the configuration in Figure $8 \mathrm{~A}$, top left, the network is connected to both the fibers and the base (Figure 8A, bottom). But since the overall fiber architecture is an array of "groves", where each grove is a set 
of closely spaced fibers assembling into a particular shape, the network can be placed either inside or outside the groves, or both. Confined to the inside, contraction and expansion generate forces that assemble and disassemble the shapes, while swelling forces imposed from the outside bend whole shapes toward or away from each other. This setup - not mutually exclusive to those above or limited to carbon nanotubes - may suggest possibilities for combined, hierarchical intra-shape and inter-shape responses, potentially each linked to a different stimulus.

\subsection{Interpretive nanostructure dances}

These basic hybrid architectures have made it possible to begin exploring how these elements communicate and function together as one collective adaptive system. What's become clear so far is that the embedded nanostructures are amazingly adept at sensing, responding to, and participating in the subtleties and complexities of the network's chemical and mechanical dynamics. As the base-attached network swells or deswells, for example, the nanostructures in the vicinity of the swelling/drying front sense even tiny asymmetries in their local force fields, so that each bends toward the drier region to an extent determined by its location along the front ${ }^{44}$ (Figure $8 \mathrm{~B}$ ). Together, they respond to the spatial and temporal progress of the transition with a sweeping wave of graded motions reflecting its slope, direction, and speed. The nanostructures can similarly sense a spatial gradient of lateral mechanical forces, as imposed when a suspended layer expands outward ${ }^{42}$. The nanostructures bend in proportion to the net force at their individual locations, with all of them bending toward the outer edges and those closest to the edge bending most (Figure 8C) - collectively translating the planar expansion and contraction into a pulsating, opening and closing 3D topographic version of the shape. They also sense differences in the location of the force along their length, bending most for lateral forces exerted at their tips.

The nanostructure array is equally adept at translating the chemical scene into a much more diverse repertoire of dynamic motions than would be possible with either component alone. The base-attached configuration is acutely sensitive to variations in local chemistry: in a $\mathrm{pH}$-sensitive polymer network, the structures bend in a current of acid and straighten where base flows ${ }^{41}$ (Figure 8D), readily adjusting when currents are switched. However, the system can alternatively be designed such that the structures are upright even when the network is contracted - and thus remain standing in homogeneous currents of either acid or base. With this modification, the hybrid now responds specifically to regions where the chemistry is changing, bending only at the boundary in the direction of the chemical gradient ${ }^{45}$ (toward the acid in Figure 8E). The directed motion results from lateral forces produced where more and less swelled regions meet: the more swelled region is able to expand laterally toward the less swelled region similar to the lateral expansion caused by raised microfeatures in Figure 4D - effectively pushing the nanostructures to bend away from it (Figure 8E, top). If the nanostructures have an asymmetric cross section, such as rectangular fins, their bending not only follows the direction of the gradient but also varies in magnitude according to its orientation.

Finer, more localized inhomogeneities in the network begin to evoke dynamic assembling shapes and patterns, linking the spatiotemporal complexities of network responses with those of nanostructure assembly down to the microscale. Responsive 
pattern formation has been studied in detail by introducing defects into the surface topography ${ }^{41}$. The nanostructures sense even a slight surface protrusion: the minor increase in local thickness generates anisotropic contraction forces (Figure 8F, inset), which drive the surrounding nanostructures to bend together around the defect into a closing microfloret (Figure 8F). By the same principle, a surface-wide landscape of inhomogeneities leads to a field of closing florets, or opening florets, small clusters, or other morphologies depending on the size, shape, and spacing of the protrusions. A single asymmetric defect further gives all the closing florets a chiral twist, producing a longrange pattern of spiraling florets each oriented based on their position relative to the defect $^{46}$ (Figure 8F). At the same time, theoretical modeling indicates that how and how much each nanostructure bends creates new local forces and surface deformations in the network $^{47}$ (Figure $8 \mathrm{G}$ ). How such feedback plays out in these complex, dynamic systems is just now on the verge of becoming amenable to experimental study. Making the question even more fascinating are the models' predictions that the nanostructures' motions influence the stimulus sensitivity of the system, suggesting that the intricate interplay between nanostructure and network motions mediates broader, more complex chemomechanical response circuits.

\section{Integrating into multicompartmental, hierarchical adaptive materials}

\subsection{Mosaic surfaces}

With so many ways to design a system, and such a diversity of responses possible with each one, the inevitable question is how to integrate a whole multi-ring circus of such systems - each pulsing with its own dynamics in response to the same or different stimuli - into a coordinated responsive symphony. The key, once again, is delineating pathways of chemical and/or mechanical communication between components. The synchronization of multiple oscillating BZ networks into a collective dynamic system provides a sophisticated example of how this can be accomplished through chemical exchange ${ }^{48,49}$. When separate compartments of reactive polymer are placed in a nonreactive polymer network, chemical signals propagate through the nonreactive medium, reaching the recipient compartment with an intensity and rate determined by the distance, catalyst concentration, and size of the compartment they came from ${ }^{48}$. Individually tailoring these factors for each compartment makes it possible to design coordinated, multipart oscillators with reactive peaks progressing in orderly fashion from one compartment to the next, with controlled timing and phase staggering (Figure 9A). Since the reactive peak leads to mechanical expansion, the surface as a whole displays a corresponding orderly progression of expanding and contracting regions that, if organized in a ring, generates wholesale rotary motion of the composite surface.

Alternatively, compartments can be pieced together like a jigsaw puzzle, such that they exchange both chemical and mechanical signals through their mutual interfaces. A surface patterned with compartments that sense different stimuli, for example, produces distinct combinatorial responses through expanding and contracting compartments pushing and pulling on each other - and generating unique position-dependent forces on embedded nanostructures ${ }^{42}$. As has been demonstrated for a compartmentalized, tipsuspended network, two interlocking $\mathrm{pH}$ - and temperature-sensitive compartments 
collectively display four different responses: at low $\mathrm{pH}$ and low temperature, low $\mathrm{pH}$ and high temperature, high $\mathrm{pH}$ and low temperature, and high $\mathrm{pH}$ and high temperature (Figure 9B). In the latter case, expansion of the $\mathrm{pH}$-responsive piece against the nonswelling temperature-responsive piece has multiple consequences: it bends its own nanostructures outward; it pushes on and further compresses the adjacent compartment; and it changes the shape of the interface, creating anisotropic forces on nanostructures located just across the border. This direct communication between compartments thus allows complex stimuli to be read out at the level of both surface-wide nanostructure patterns and individual nanostructure bending profiles. The compartments may also potentially influence each other's chemical responses through the imposed stresses, analogously to the mechanisms discussed in section 2.3.

\subsection{Super chemo-mechano-chemical feedback systems}

Yet the most telling way to communicate one compartment's complex interior spatiotemporal dynamics to another compartment is through the continuously reconfiguring topographic patterns at its surface. In this spirit, the pulsing surface deformations of the BZ network have been shown to translate the interior reaction kinetics into external flows and currents that in turn mix the components of an overlying medium ${ }^{7}$. The swelling-induced creasing discussed in section 3.2 has even been used to manipulate specific biochemical reactions, by hiding and exposing a surface-attached enzyme as the creases form and unform ${ }^{33}$.

One recently developed system - based on the hybrid architectures combined with the fluidic compartmentalization found in many protocell models ${ }^{50}-$ not only shows how such surface dynamics can be brought to bear in a bona fide multicompartmental material, but also demonstrates how the motions of reconfiguring nanostructures can be used to translate the detailed chemomechanical processes in one compartment into adaptive, completely separate but intricately connected chemical responses in another ${ }^{51}$. The material is organized as a bilayer: one layer consists of the polymer network and its solvent, and the other is a thin, overlying fluid compartment containing a distinct set of chemical reagents that don't mix with the first layer. The nanostructures have catalysts attached to their tips, so that as they bend and dance in accord with the first layer, their tips bring the catalysts into and out of the second layer - inducing and shaping the kinetics, duration, and spatial profile of new chemical reactions (Figure 9C, top). Since the polymer chains and the reagents never see each other, their chemistries can be chosen independently; the only link between them is the mechanical dynamics of the nanostructures that reversibly connect the two compartments.

However, the separate chemistries also make the system ripe for higher order chemo-mechano-chemical feedback. The chemistry in the second layer can potentially influence tip assembly and dynamics, but the reactions can also be designed to produce the heat, light, pressure, or other stimuli that induce polymer reconfigurations in the first place. A complete feedback loop has indeed been created, by pairing a temperatureresponsive polymer network in the first layer with a heat-generating reaction in the second $^{51}$ (Figure 9C, bottom). As cold temperatures swell the network, the nanostructures straighten, enter the second layer, and turn on the heating reaction; the heat causes the polymer network to contract, bend the nanostructures, withdraw the catalyst, and turn off 
the reaction - until the temperature falls below the set point and restarts the cycle. The dynamics of the chemical exchange between the two compartments is highly sensitive to the mechanical details of the nanostructure motions. In this way, the system's internal chemomechanical feedback dynamics become part of a bigger chemo-mechano-chemical feedback loop that includes the stimulus as an integral part. The layered architecture lends itself equally well to further feedback with additional polymer compartments or with the surrounding environment.

\section{Outlook}

Chemomechanical feedback is as inherent to synthetic materials as it is to living systems - when cultivated as a design resource, it produces a diversity of unique, dynamic processes, from traveling waves and topographic patterning, to chemicallycoupled surface buckling, to spiraling nanostructures, to motion-triggered reactions. Each realm continues to grow in surprising and promising directions, but the greatest adaptive possibilities will undoubtedly come from exploring the many pathways connecting them. Polymer chemistries provide an increasing range of stimulus sensitivities, but, at the same time, the induced responses are all essentially reaction-diffusion processes - with built-in feedback between propagating molecular and mechanical changes - such that designing physical architectures and kinetics in tandem, as has been done for known reactiondiffusion systems in polymer networks, may open routes to programming complex 3D spatiotemporal responses. Surface-wide buckling connects with this picture in at least two ways: it not only superimposes large-scale architectures on the system but also, given its sensitivity to diffusion kinetics and geometry, will most likely be responsive to complex chemical dynamics. Nanostructures assembling over multiple scales directly contact both the surface and the interior of the matrix, and may be in a position to integrate wrinkling and reaction-diffusion dynamics in unforeseen ways. New reactions triggered in an adjacent compartment may in turn influence not only polymer responses but also surface instabilities and nanostructure interactions. The possibilities are endless - the hybrid and multicompartmental systems we've discussed here provide a unifying platform for bringing all of these processes together and building on their connections.

Yet the potential sources of chemomechanical feedback extend well beyond these systems. While less explored from this perspective, a balance between molecular interactions and mechanical stress has been suggested to shape crystal formation on organic templates ${ }^{52}$; interfacial tension may influence the growth of nanoparticles in solution $^{53}$; and the growth of inverse opals entails both mechanical and chemical exchange between assembling colloids and the matrix ${ }^{54}$. Catalysis and chirality can be dynamically controlled by mechanical rotation of a molecular motor ${ }^{55}$, and soft robots ${ }^{56}$, liquid crystals and supramolecular polymers ${ }^{57}$ provide unique classes of mechanochemistry. As the systems in this review have taught us, the key to incorporating such phenomena into the design of responsive materials lies in looking not only at the contributions of chemistry and mechanics to a stable equilibrium state, but at the iterative, step-by-step dynamics of the exchange between them. In any system, chemical and mechanical changes rarely take place on the same time scale - they invariably evolve through back-and-forth responsive feedback, and, as we've seen both here and in living 
organisms, it is this intrinsically adaptive behavior that provides the most basic seeds for constructing higher order, sophisticated adaptive responses.

What's more, multiscale patterns of chemistry and topography underlie just about every functional property of a material: from its color, wettability, and adhesion, to manipulation of light, heat, and electric current, to the control of ice formation, bacterial colonization, and cell differentiation on its surface. Fine-tuned adaptations of any one property, let alone several, can be a painstaking matter of coordinating precise molecular and structural adjustments, but the interplay between chemistry and mechanics does the work for us - they shape each other. With infinitely different variations emerging from the self-generating interplay among different time scales, size scales, and forms of energy, the answer to whether synthetic materials can ever approach the multiresponsive, multifunctional, hierarchical complexity of biological materials appears to be: there's no reason why not. Bringing out the fundamental material capacity for chemomechanical feedback enables us to envision a nearly unlimited variety of self-adapting systems, from microscale biomedical devices up through entire self-regulating buildings, bridges, and transport systems - each built from layer upon layer of dynamic adaptive behaviors selfintegrating from the bottom up.

\section{Acknowledgements}

This work was supported by the U.S. Department of Energy, Office of Basic Energy Sciences, Division of Materials Sciences and Engineering under award number DESC0005247 (design of adaptive materials); by the U.S. Air Force Office of Scientific Research Multidisciplinary University Research Initiative under award number FA955009-1-0669-DOD35CAP (dynamic optical structures); and by the U.S. National Science Foundation under award number CMMI-1124839 (chemo-mechanical feedback systems). We gratefully acknowledge Dr. Michael DeVolder for providing the image of hydrogelinfiltrated carbon nanotubes.

\section{References}

1. D. Kuckling, Colloid and Polymer Science, 2009, 287, 881-891.

2. I. Tokarev, M. Motornov, S. Minko, Journal of Materials Chemistry, 2009, 19, 6932.

3. M. A. C. Stuart, W. T. S. Huck, J. Genzer, M. Muller, C. Ober, M. Stamm, G. B. Sukhorukov, I. Szleifer, V. V. Tsukruk, M. Urban, F. Winnik, S. Zauscher, I. Luzinov, S. Minko, Nat Mater, 2010, 9, 101-113.

4. T. Tanaka, D. J. Fillmore, The Journal of Chemical Physics, 1979, 70, 1214.

5. C. J. Campbell, R. Klajn, M. Fialkowski, B. A. Grzybowski, Langmuir, 2005, 21, 418423. 
6. S. K. Smoukov, A. Bitner, C. J. Campbell, K. Kandere-Grzybowska, B. A. Grzybowski, J Am Chem Soc, 2005, 127, 17803-17807.

7. R. Yoshida, Adv Mater, 2010, 22, 3463-83.

8. V. V. Yashin, O. Kuksenok, P. Dayal, A. C. Balazs, Rep Prog Phys, 2012, 75, 066601.

9. P. X. Yuan, O. Kuksenok, D. E. Gross, A. C. Balazs, J. S. Moore, R. G. Nuzzo, Soft Matter, 2013, 9, 1231-1243.

10. I. C. Chen, O. Kuksenok, V. V. Yashin, R. M. Moslin, A. C. Balazs, K. J. Van Vliet, Soft Matter, 2011, 7, 3141.

11. R. Yoshida, M. Tanaka, S. Onodera, T. Yamaguchi, E. Kokufuta, J Phys Chem A, 2000, 104, 7549-7555.

12. S. K. De, N. R. Aluru, B. Johnson, W. C. Crone, D. J. Beebe, J. Moore, J Microelectromech S, 2002, 11, 544-555.

13. H. G. Schild, Prog Polym Sci, 1992, 17, 163-249.

14. S. Ramakrishnan, V. Gopalakrishnan, C. F. Zukoski, Langmuir, 2005, 21, 9917 9925.

15. K. Sumaru, M. Kameda, T. Kanamori, T. Shinbo, Macromolecules, 2004, 37, 49494955.

16. T. Satoh, K. Sumaru, T. Takagi, T. Kanamori, Soft Matter, 2011, 7, 8030.

17. A. Matsumoto, T. Kurata, D. Shiino, K. Kataoka, Macromolecules, 2004, 37, 15021510 .

18. R. M. Luo, H. Li, E. Birgersson, K. Y. Lam, J Biomed Mater Res A, 2008, 85A, 248257.

19. C. M. Kingsbury, P. A. May, D. A. Davis, S. R. White, J. S. Moore, N. R. Sottos, Journal of Materials Chemistry, 2011, 21, 8381.

20. E. C. Cho, J. Lee, K. Cho, Macromolecules, 2003, 36, 9929-9934.

21. J. Horváth, I. Szalai, J. Boissonade, P. De Kepper, Soft Matter, 2011, 7, 8462.

22. A. Suzuki, T. Ishii, J Chem Phys, 1999, 110, 2289-2296.

23. W. Hong, Z. Liu, Z. Suo, International Journal of Solids and Structures, 2009, 46, 3282-3289. 
24. S. Cai, Z. Suo, Journal of the Mechanics and Physics of Solids, 2011, 59, 2259-2278.

25. T. Tanaka, S. T. Sun, Y. Hirokawa, S. Katayama, J. Kucera, Y. Hirose, T. Amiya, Nature, 1987, 325, 796-798.

26. S. Yang, K. Khare, P.-C. Lin, Advanced Functional Materials, 2010, 20, 2550-2564.

27. B. Li, Y.-P. Cao, X.-Q. Feng, H. Gao, Soft Matter, 2012, 8, 5728.

28. H. Vandeparre, P. Damman, Physical Review Letters, 2008, 101.

29. J. Y. Chung, A. J. Nolte, C. M. Stafford, Adv Mater, 2009, 21, 1358-1362.

30. H. Vandeparre, S. Gabriele, F. Brau, C. Gay, K. K. Parker, P. Damman, Soft Matter, 2010, 6, 5751.

31. M. Guvendiren, J. A. Burdick, S. Yang, Soft Matter, 2010, 6, 5795.

32. M. K. Kang, R. Huang, Soft Matter, 2010, 6, 5736.

33. J. Kim, J. Yoon, R. C. Hayward, Nat Mater, 2010, 9, 159-164.

34. S. Saha, D. Copic, S. Bhaskar, N. Clay, A. Donini, A. J. Hart, J. Lahann, Angew Chem Int Ed Engl, 2012, 51, 660-5.

35. L. Hsu, C. Weder, S. J. Rowan, Journal of Materials Chemistry, 2011, 21, 2812.

36. K. L. Dagnon, K. Shanmuganathan, C. Weder, S. J. Rowan, Macromolecules, 2012, 45, 4707-4715.

37. A. E. Way, L. Hsu, K. Shanmuganathan, C. Weder, S. J. Rowan, ACS Macro Letters, 2012, 1, 1001-1006.

38. M. De Volder, S. H. Tawfick, S. J. Park, D. Copic, Z. Zhao, W. Lu, A. J. Hart, Adv Mater, 2010, 22, 4384-9.

39. A. Grinthal, S. H. Kang, A. K. Epstein, M. Aizenberg, M. Khan, J. Aizenberg, Nano Today, 2012, 7, 35-52.

40. R. Klajn, J. F. Stoddart, B. A. Grzybowski, Chem Soc Rev, 2010, 39, 2203-37.

41. P. Kim, L. D. Zarzar, X. M. He, A. Grinthal, J. Aizenberg, Curr Opin Solid St M, 2011, 15, 236-245. 
42. L. D. Zarzar, P. Kim, M. Kolle, J. Brinker, J. Aizenberg, B. Kaehr, Angew Chem Int Edit, 2011, 50, 9356-9360.

43. M. De Volder, S. H. Tawfick, D. Copic, A. J. Hart, Soft Matter, 2011, 7, 9844.

44. A. Sidorenko, T. Krupenkin, A. Taylor, P. Fratzl, J. Aizenberg, Science, 2007, 315, 487-490.

45. L. D. Zarzar, Q. H. Liu, X. M. He, Y. H. Hu, Z. G. Suo, J. Aizenberg, Soft Matter, 2012, 8, 8289-8293.

46. P. Kim, L. D. Zarzar, X. H. Zhao, A. Sidorenko, J. Aizenberg, Soft Matter, 2010, 6, 750-755.

47. W. H. Wong, T. F. Guo, Y. W. Zhang, L. Cheng, International Journal of Solids and Structures, 2010, 47, 2034-2042.

48. V. V. Yashin, S. Suzuki, R. Yoshida, A. C. Balazs, Journal of Materials Chemistry, 2012, 22, 13625.

49. M. L. Smith, C. Slone, K. Heitfeld, R. A. Vaia, Advanced Functional Materials, 2013, in press.

50. A. J. Dzieciol, S. Mann, Chem Soc Rev, 2012, 41, 79-85.

51. X. M. He, M. Aizenberg, O. Kuksenok, L. D. Zarzar, A. Shastri, A. C. Balazs, J. Aizenberg, Nature, 2012, 487, 214-218.

52. T. Y. J. Han, J. Aizenberg, Chem Mater, 2008, 20, 1064-1068.

53. J. M. Garcia-Ruiz, E. Melero-Garcia, S. T. Hyde, Science, 2009, 323, 362-365.

54. B. Hatton, L. Mishchenko, S. Davis, K. H. Sandhage, J. Aizenberg, P Natl Acad Sci USA, 2010, 107, 10354-10359.

55. J. B. Wang, B. L. Feringa, Science, 2011, 331, 1429-1432.

56. F. Ilievski, A. D. Mazzeo, R. E. Shepherd, X. Chen, G. M. Whitesides, Angew Chem Int Edit, 2011, 50, 1890-1895.

57. T. Aida, E. W. Meijer, S. I. Stupp, Science, 2012, 335, 813-817.

58. O. Kuksenok, V. V. Yashin, A. C. Balazs, Soft Matter, 2009, 5, 1835. 
Figure 1

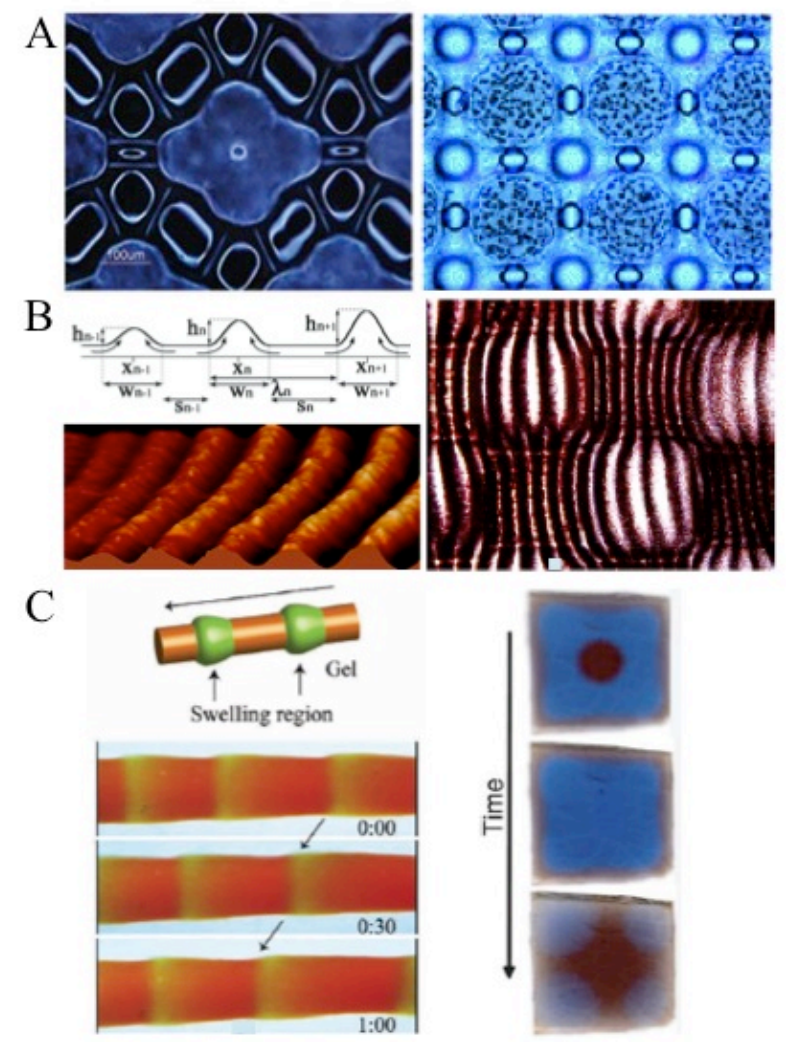


Figure 2

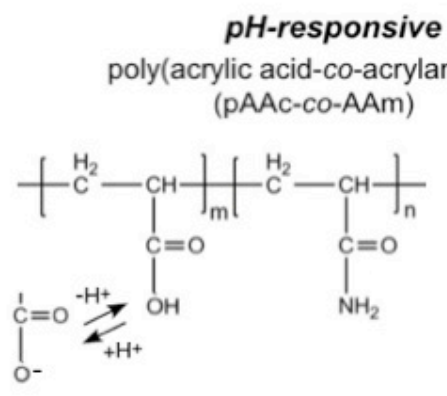

pH-responsive

(pAAc-CO-AAm)

Glucose-responsive

phenylborate modified pNIPAAm

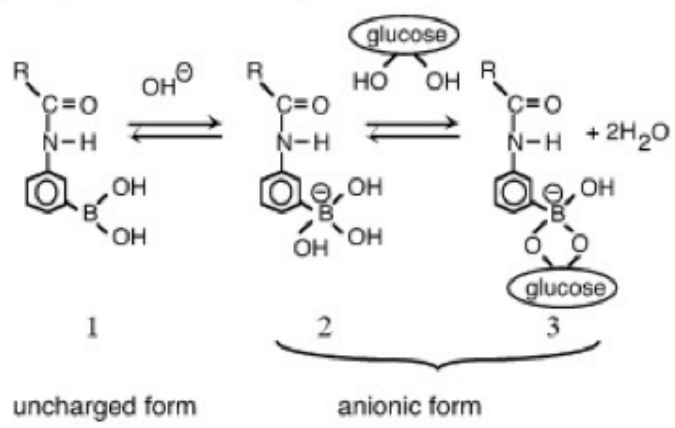

Temperature-responsive

poly( $\mathrm{N}$-isopropylacrylamide)

(pNIPAAm)

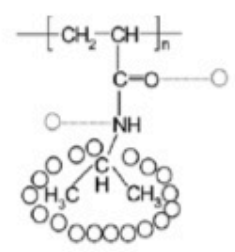

Light-, temperature-, and pH-responsive

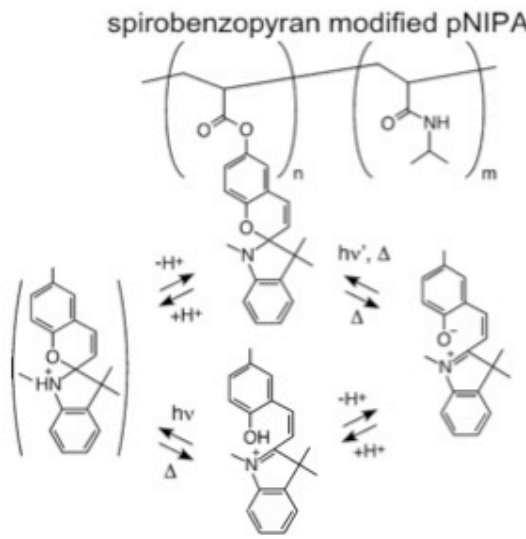

Figure 3

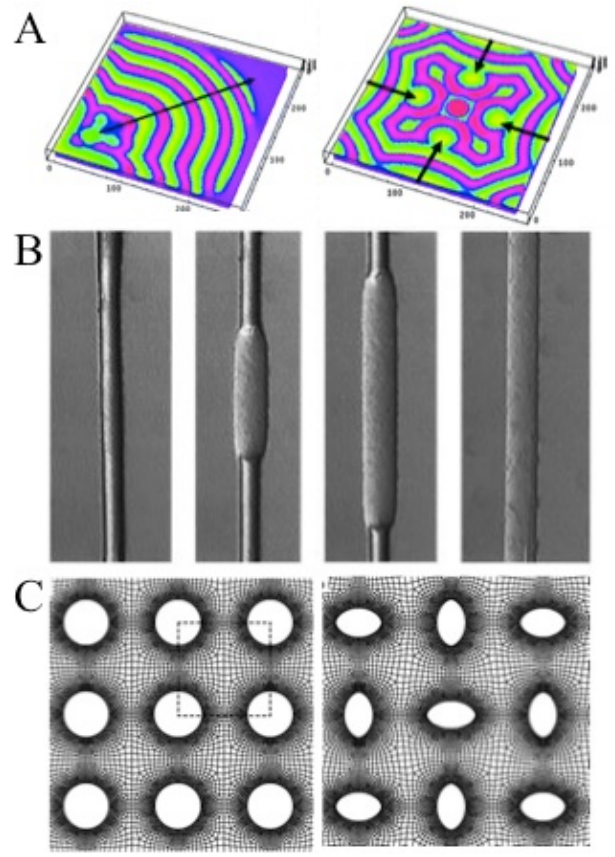


Figure 4
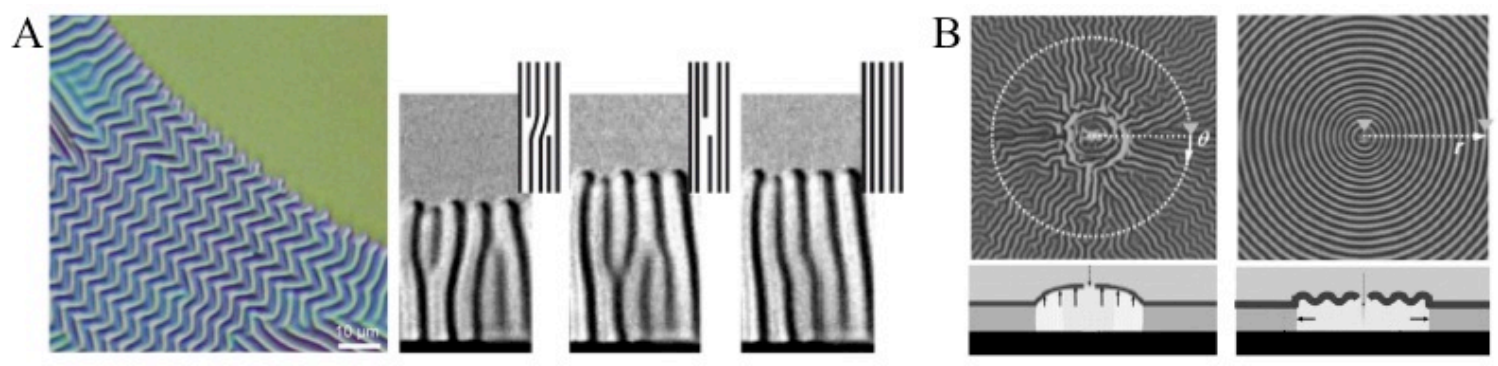

C

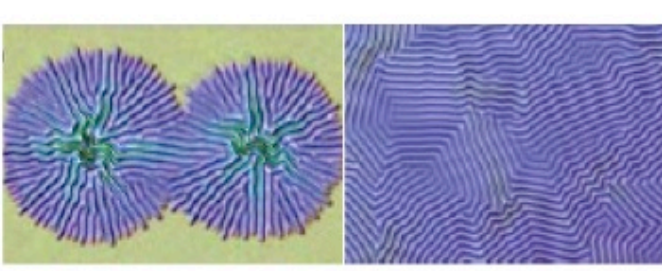

D

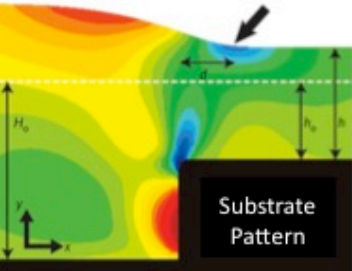

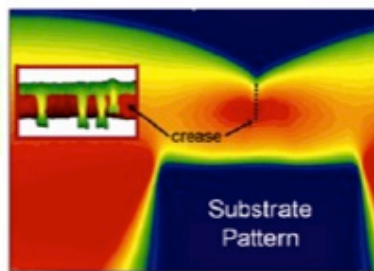

Figure 5
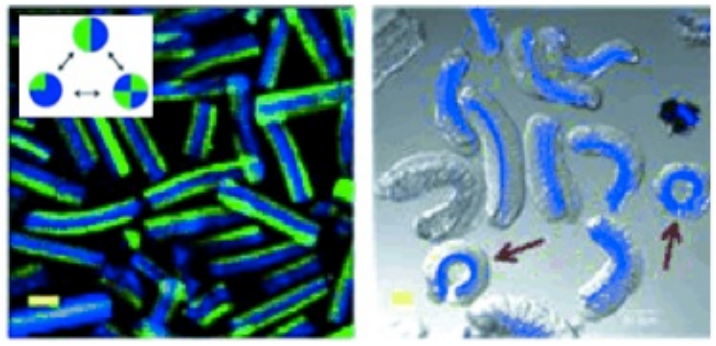

Figure 6

A

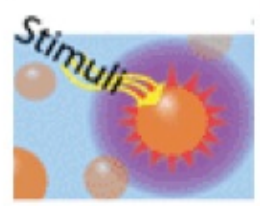

B

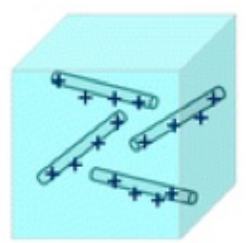

Fiber interactions "off"
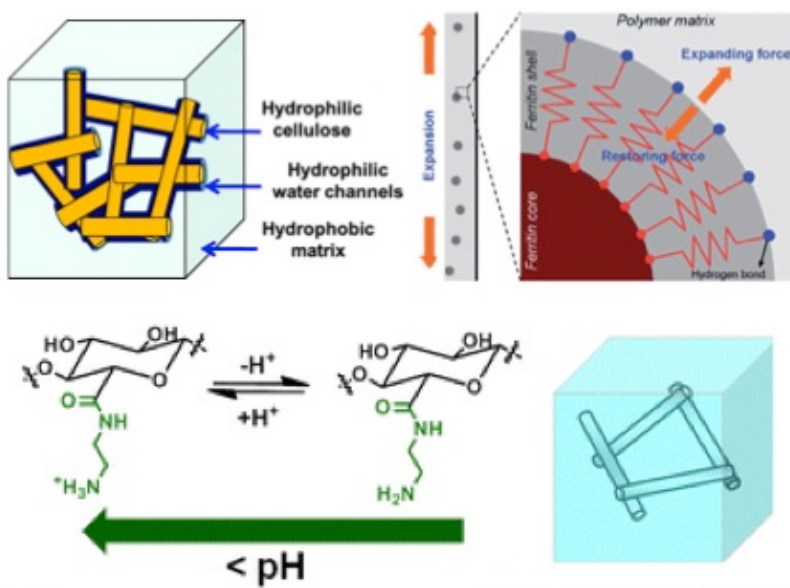

Fiber interactions "on" 
Figure 7
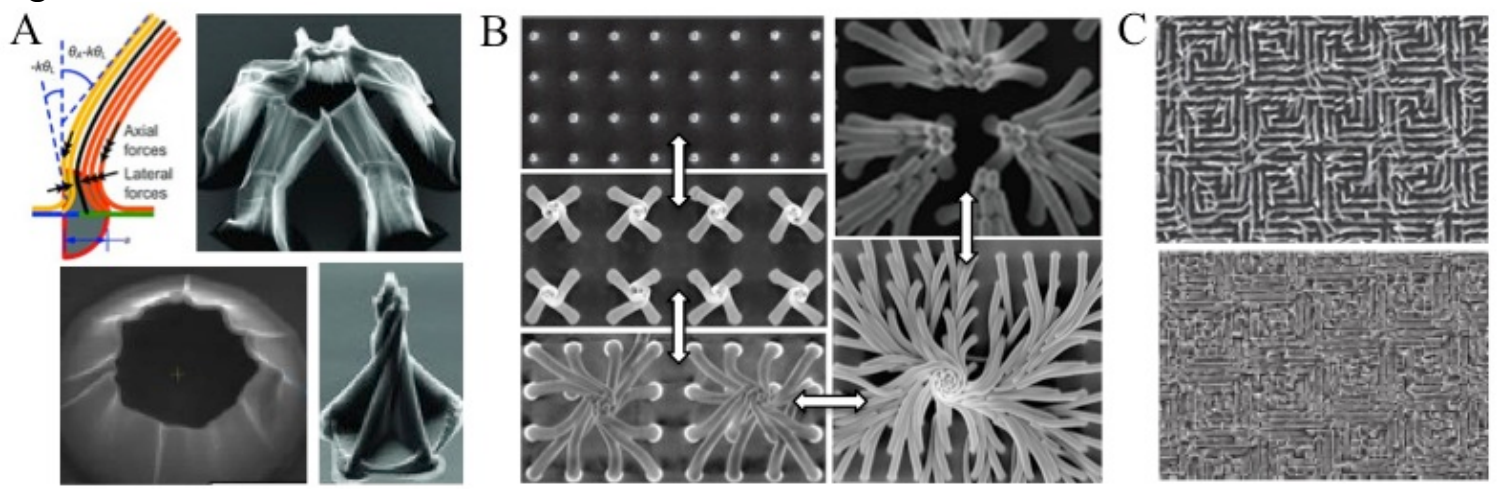

Figure 8
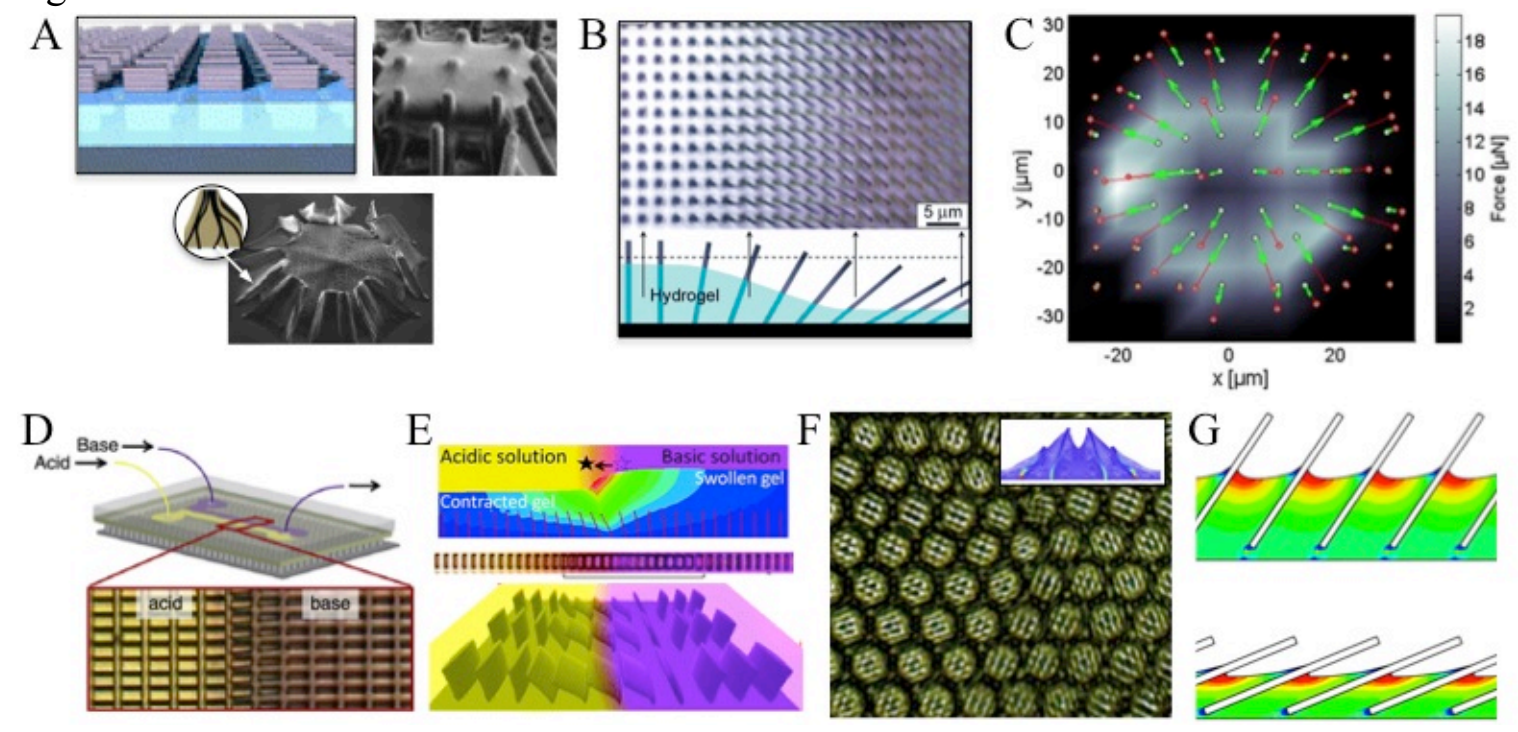

Figure 9
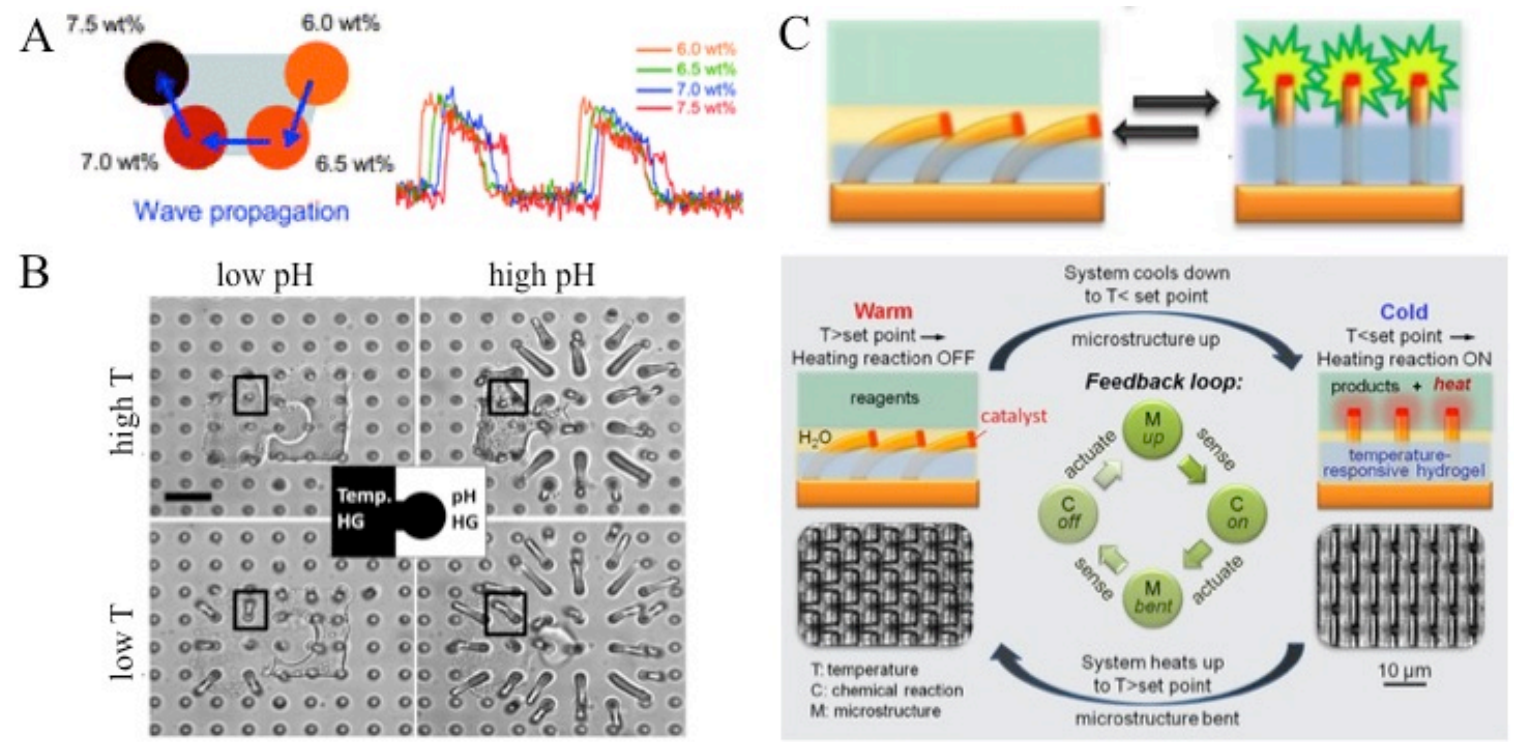


\section{Figure captions}

Figure 1. Coupling chemical reactions and elastic reconfiguration. A. Reactions propagating inside a polymer network sculpt the network into 3D topographic patterns. Left and right differ in the geometry of the diffusion fronts. B. Shape, size, and spacing of each pattern feature depend on both chemical and mechanical conditions. Right: curvature, size, and spacing adjust to local variations in polymer crosslinking density. C. Reversible reactions generate travelling surface waves and dynamic shape changes that track the spatiotemporal progress of the reaction. [Adapted with permission from ref ${ }^{5}$ $(\mathrm{A}), \operatorname{ref}^{6}(\mathrm{~B}), \operatorname{ref}^{7}(\mathrm{C}$, left $), \operatorname{ref}^{10}(\mathrm{C}$, right $\left.)\right]$

Figure 2. Reactive groups on the polymer chains link a diversity of stimuli with a diversity of mechanisms for interweaving chemical responses with mechanical dynamics. [Adapted with permission from ref $^{41},{ }^{20},{ }^{15},{ }^{17}$ ]

Figure 3. Patterning chemical dynamics with mechanical stress. A. Local stresses induce and shape reaction propagation pathways. B. Global stress triggers the response of a temperature-sensitive network; the response is proportional to the stress magnitude (increasing left to right). C. Patterned defects generate anisotropic stress fields, solvent and chemical concentrations, and network deformations. [Adapted with permission from $\left.\operatorname{ref}^{58}(\mathrm{~A}),{ }^{22}(\mathrm{~B}),{ }^{23}(\mathrm{C})\right]$

Figure 4. Diffusion-coupled surface buckling. A. Expansion of the polymer network generates surface instabilities and buckling; collective wrinkling patterns grow and change as the diffusion front propagates. B. Different diffusion kinetics (fast, left; slow, right) create different patterns. C. Colliding patterns propagating from different surface defects reconfigure into new collective morphologies. D. Patterned substrate defects influence buckling morphology and location (left, instability develops at arrow); creasing influences further chemical and diffusion processes by altering the local polymer density (right, density highest at arrow). [Adapted with permission from $\operatorname{ref}^{28}(\mathrm{~A}),{ }^{29}(\mathrm{~B}),{ }^{30}(\mathrm{C})$, $\left.{ }^{33}(\mathrm{D})\right]$

Figure 5. Buckling at gel-microstructure interfaces. Hybrid microcylinders (left: swellable polymer in green; nonswellable elastic structure in blue; inset shows cross sections of different hybrid configurations) bend, curl, and twist (right; arrows highlight twisting) as swelling creates interfacial stresses. [Adapted with permission from ref ${ }^{34}$ ]

Figure 6. Responsive micro/nanostructures embedded in polymer networks. A. Nanostructures can induce and channel chemical responses, diffusion, and/or mechanical stress inside a responsive matrix. B. The nanostructures can be designed to alter their associations and function in response to dynamic matrix conditions. [Adapted with permission from $\left.\operatorname{ref}^{35},{ }^{36}(\mathrm{~A}) ;{ }^{37}(\mathrm{~B})\right]$ 
Figure 7. Chemomechanical patterning of anchored, oriented nanostructures. A. Anisotropic forces and interfiber interactions shape carbon nanotubes into bridged, iris, helical, and other configurations. B. The interplay between mechanical and chemical forces reversibly organizes flexible nanofibers into diverse chiral structures through hierarchical stages of assembly and disassembly. C. Dynamic feedback between assembling nanostructures and solvent creates long-range patterns. [Adapted with permission from $\left.\operatorname{ref}^{38}(\mathrm{~A}),{ }^{39}(\mathrm{~B}, \mathrm{C})\right]$

Figure 8. Chemomechanical feedback in hybrid polymer network-nanostructure systems. A. Hybrid configurations: polymer network attached to both substrate base and sides of nanostructures (top left); suspended polymer layer (top right); polymer within and/or between carbon nanotube clusters. B. Nanostructure motions are sensitive to fine gradients in swelling at a solvent front; (C) to gradients in force magnitude and direction induced by anisotropic expansion; (D) to spatial chemical patterning; (E) to location and direction of chemical gradients, and (F) to variations in surface topography. G.

Nanostructure motions induce mechanical stresses and associated chemical changes in the polymer network. [Adapted with permission from $\operatorname{ref}^{45}(\mathrm{~A}, \mathrm{E}),{ }^{42}(\mathrm{~A}, \mathrm{~B}),{ }^{44}(\mathrm{~B}),{ }^{46}(\mathrm{~F})$, $\left.{ }^{47}(\mathrm{G})\right]$

Figure 9. Hierarchical feedback in multicompartmental materials. A. Reactive patches in a nonreactive matrix generate collective chemical waves and synchronized expansion/contraction. B. Interlocking patches with different stimulus sensitivities create unique combinatorial responses for different stimulus combinations. C. Hybrid systems incorporated into a bilayer material generate higher order chemo-mechano-chemical responses: catalyst-bearing micro/nanostructure tips reversibly turn on and off new chemical reactions as gel-driven bending moves them into and out of a reagent layer (top). Coordinating reaction outputs with polymer stimulus sensitivity creates chemomechano-chemical feedback loops (bottom). [Adapted with permission from $\operatorname{ref}^{48}(\mathrm{~A})$, $\left.{ }^{42}(\mathrm{~B}),{ }^{51}(\mathrm{C})\right]$ 